\title{
Overview of the status/results in the exotics sector
}

\author{
Chengping Shen ${ }^{1, \star}$ and Changsheng $\mathrm{Li}^{1}$ for the Belle Collaboration \\ ${ }^{1}$ School of Physics and Nuclear Energy Engineering, Beihang University, Beijing, People's Republic of China
}

\begin{abstract}
Many exotic hadronic states beyond the conventional quark model (called charmoniumlike/bottomoniumlike states or $X Y Z$ particles) were found in the $B$-factories or energy scans of the cross sections of $e^{+} e^{-}$annhilation into conventional quarkonia and light hadrons. Their nature properties were proposed including glueballs, hybrids, multiquark states, hadron molecules, etc. Dramatic progress was made in the study of the them after the running of the $B$-factories. In this report, I present the most recent results on the $X Y Z$ results from Belle experiment.
\end{abstract}

\section{Introduction}

During the past thirteen years many exotic states decaying into conventional quarkonia and light hadrons, the so-called " $X Y Z$ " particles, have been reported in many different reactions [1]. They correspond to high excitations and have masses above the $D \bar{D}$ or $B \bar{B}$ thresholds. Most cannot be described well by quarkonium potential models [1-3]. Their unusual properties have stimulated considerable theoretical interest and various interpretations have been proposed, including tetraquarks, molecules, hybrids, or hadrocharmonia [1, 3, 4]. To distinguish among these explanations, more experimental information is needed. Here, I present the most recent results on the study of the XYZ states from the Belle experiment.

\section{The $X$ states}

The observation of the $X(3872)$ [5] in 2003 kicked off the door of searching for exotic states in experiment. Considerable efforts by both experimentalists and theorists have been invested to clarify its nature. As a result, we know precisely its mass $(3871.69 \pm 0.17) \mathrm{MeV} / c^{2}$, have a stringent limit on its width (less than $1.2 \mathrm{MeV}$ at $90 \%$ confidence level (C.L.)) and have a definitive $J^{P C}$ assignment of $1^{++}$. Due to the close mass to $D^{* 0} \bar{D}^{0}$ threshold, it was a considerable speculation from the beginning that the $X(3872)$ might be a molecule-like bound state of $D^{* 0}$ and $\bar{D}^{0}$ meson. The generally accepted interpretation at present for the still-fascinating $X(3872)$ is a mixture of a charmonium state $\chi_{c 1}(2 P)$ and an $S$-wave $D^{* 0} \bar{D}^{0}$ molecule. More experimental information on the production and decays of the $X(3872)$ will shed additional light on its nature.

New interesting information on the $X(3872)$ is from BESIII by measuring the process $e^{+} e^{-} \rightarrow$ $\gamma X(3872) \rightarrow \gamma \pi^{+} \pi^{-} J / \psi$ at $e^{+} e^{-}$center-of-mass energies from $\sqrt{s}=4.009 \mathrm{GeV}$ to $4.420 \mathrm{GeV}$ [6]. The observed statistical significance of $X(3872)$ in the $M\left(\pi^{+} \pi^{-} J / \psi\right)$ distribution reaches $6.3 \sigma$. Figure 1(a)

^e-mail: shencp@buaa.edu.cn 
shows the energy-dependent cross section together with the fitted results with a $Y(4260)$ resonance, a linear continuum, or a $E 1$-transition phase space term. The $Y(4260)$ resonance describes the data better than the other two options, which strongly supports the existence of the radiative transition process $Y(4260) \rightarrow \gamma X(3872)$. This is the first time that we observe a relation between the $X$ and $Y$ states.

It is natural to search for a similar state $X_{b}$ with $J^{P C}=1^{++}$in the bottomonium system. The search for $X_{b}$ supplies important information about the discrimination of a compact multiquark configuration and a loosely bound hadronic molecule configuration for the $X(3872)$. The CMS Collaboration ever reported a null search for such a state in the $\pi^{+} \pi^{-} \Upsilon(1 S)$ final state [7]. However, unlike the $X(3872)$, whose decays exhibit large isospin violation, the $X_{b}$ would decay preferably into $\pi^{+} \pi^{-} \pi^{0} \Upsilon(1 S)$ rather than $\pi^{+} \pi^{-} \Upsilon(1 S)$ if it exists. So Belle did a search for an $X_{b}$ signal decaying to $\omega \Upsilon(1 S)$ in $e^{+} e^{-} \rightarrow$ $\gamma X_{b} \rightarrow \gamma \pi^{+} \pi^{-} \pi^{0} \Upsilon(1 S)$ at a center-of-mass energy of $10.867 \mathrm{GeV}$ based on a $118 \mathrm{fb}^{-1}$ data sample [8].

Figure 1(b) shows the final $\omega \Upsilon(1 S)$ invariant mass distribution with the requirement of $M\left(\pi^{+} \pi^{-} \pi^{0}\right)$ within the $\omega$ signal region; the $X_{b}$ is searched for from 10.55 to $10.65 \mathrm{GeV} / c^{2}$. The dots with error bars are from data, the solid histogram is from the normalized contribution of $e^{+} e^{-} \rightarrow \omega \chi_{b J}(J=$ $0,1,2)$ and the blank histogram is from the normalized $\omega$ mass sideband, defined as $0.54 \mathrm{GeV} / c^{2}<$ $M\left(\pi^{+} \pi^{-} \pi^{0}\right)<0.72 \mathrm{GeV} / c^{2}$. No obvious $X_{b}$ signal is observed. The upper limit on the yield of the $X_{b}$ signal events is 4.0 at $90 \%$ C.L. with a mass at $10.6 \mathrm{GeV} / c^{2}$. The product branching fraction is determined to be $\mathcal{B}\left(\Upsilon(5 S) \rightarrow \gamma X_{b}\right) \mathcal{B}\left(X_{b} \rightarrow \omega \Upsilon(1 S)\right)<2.9 \times 10^{-5}$ at $90 \%$ C.L.
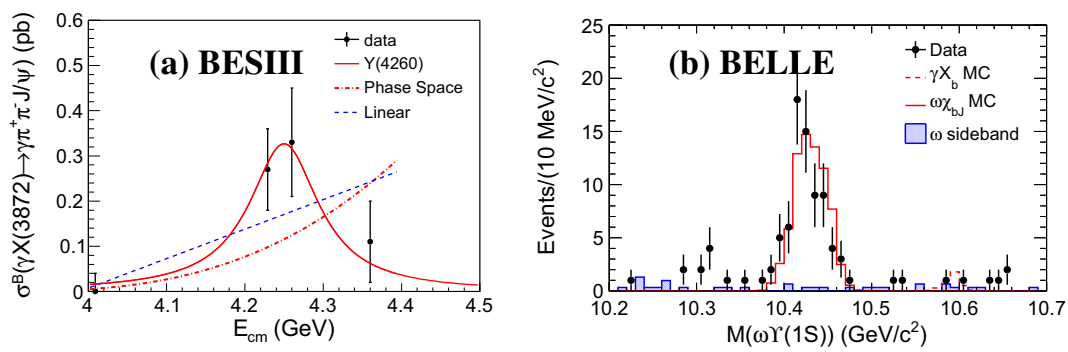

Figure 1. Results on the $X(3872)$ and its similar state in the bottomonium system $X_{b}$ from BESIII and Belle. (a) The $\sigma^{B}\left[e^{+} e^{-} \rightarrow \gamma X(3872)\right] \times \mathcal{B}\left[X(3872) \rightarrow \pi^{+} \pi^{-} J / \psi\right]$ distribution and fit results with different line shapes from BESIII; (b) The $\omega \Upsilon(1 S)$ invariant mass distribution in $e^{+} e^{-} \rightarrow \gamma \omega \Upsilon(1 S)$ at $\sqrt{s}=10.867 \mathrm{GeV}$ from Belle measurement.

\section{The $Y$ states}

States with $J^{P C}=1^{--}$can be studied with initial state radiation (ISR) in Belle's and BaBar's large $\Upsilon(4 S)$ data samples or via direct production in $e^{+} e^{-}$collisions at BESIII.

The $Y(4260)$ state was first observed by BaBar in $e^{+} e^{-} \rightarrow \pi^{+} \pi^{-} J / \psi$ [9] and this was confirmed by the CLEO [10] and Belle experiments [11] with the same technique. Moreover, Belle reported a broad structure near 4.0 GeV called $Y(4008)$ [12]. Later, the BaBar Collaboration reported updated results with a $454 \mathrm{fb}^{-1}$ data sample and a modified approach for the background description [13]; the $Y(4260)$ state was observed with improved significance, but the $Y(4008)$ structure was not confirmed. Instead, they attributed the structure below the $Y(4260)$ to exponentially falling non-resonant $\pi^{+} \pi^{-} J / \psi$ production. In 2013, Belle updated $e^{+} e^{-} \rightarrow \pi^{+} \pi^{-} J / \psi$ process again with a $967 \mathrm{fb}^{-1}$ data sample. Not only the $Y(4260)$ state was observed clearly, but also the Y(4008) was confirmed [14]. 
A better understanding of the structures observed in $e^{+} e^{-} \rightarrow \pi^{+} \pi^{-} J / \psi$ would benefit from improved measurements in the future.

In an analysis of the $e^{+} e^{-} \rightarrow \pi^{+} \pi^{-} \psi(2 S)$ process, BaBar found a structure near $4.32 \mathrm{GeV} / c^{2}$ [15], while Belle observed two resonant structures at 4.36 and $4.66 \mathrm{GeV} / c^{2}$ [16]. The latest $e^{+} e^{-} \rightarrow$ $\pi^{+} \pi^{-} \psi(2 S)$ analysis from the BaBar experiment with its full data sample confirmed the existence of the $Y(4660)$ state [17]. To characterize more precisely the properties of the $Y(4360)$ and $Y(4660)$, Belle updated the $e^{+} e^{-} \rightarrow \pi^{+} \pi^{-} \psi(2 S)$ process with the full Belle data $980 \mathrm{fb}^{-1}$ [18]. Figure 2 shows the invariant mass distribution of $\pi^{+} \pi^{-} \psi(2 S)$ from the updated measurement. Besides the $Y(4360)$ and $Y(4660)$, there are a few events in the vicinity of the $Y(4260)$ mass, so an alternative fit with a coherent sum of $Y(4260), Y(4360)$, and $Y(4660)$ amplitudes was performed as shown in Fig. 2. But the signal significance of the $Y(4260)$ is only $2.4 \sigma$. With more statistic at BelleII, it will be clear if the $Y(4260)$ can decay to $\pi^{+} \pi^{-} \psi(2 S)$.
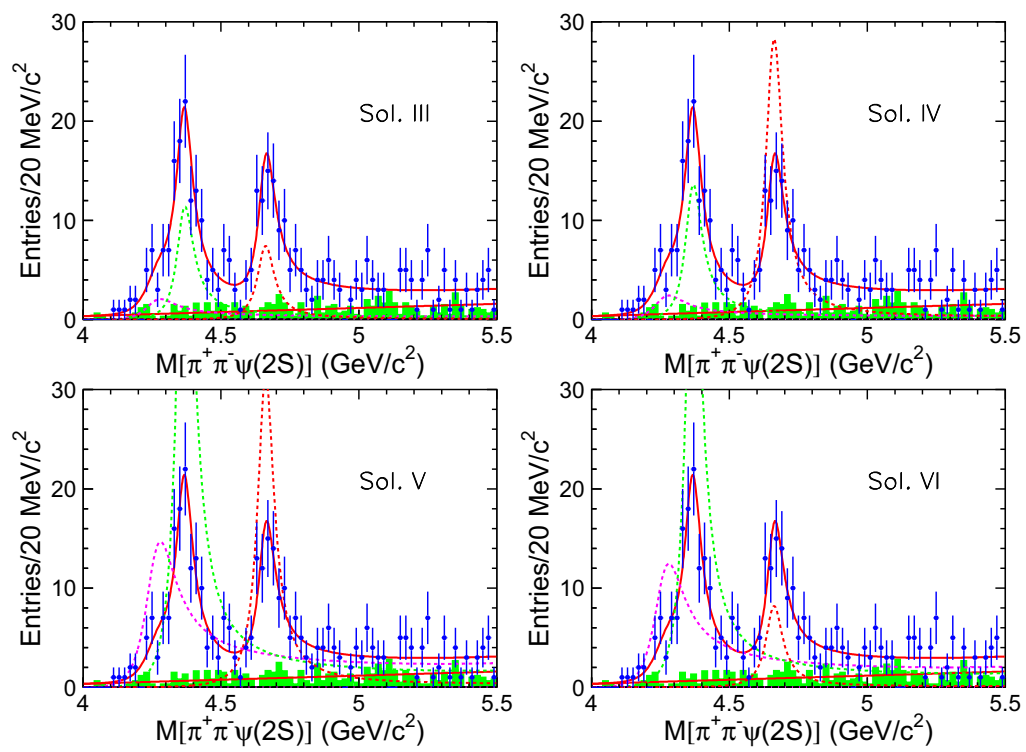

Figure 2. The four solutions from the fit to the $\pi^{+} \pi^{-} \psi(2 S)$ invariant mass spectra with the $Y(4260)$ included. The curves show the best fit and the dashed curves show the contributions from the three Breit-Wigner components.

BESIII accumulated large data samples between 4.0 and $4.6 \mathrm{GeV}$ for the study of the charmoniumlike states. With these data samples, BESIII measured some interesting processes, for examples, $e^{+} e^{-} \rightarrow \pi^{+} \pi^{-} h_{c}$ [19], $\omega \chi_{c 0}$ [20], $\pi^{+} \pi^{-} \psi_{2}$ [21]. In the future BelleII needs to check $e^{+} e^{-} \rightarrow \pi^{+} \pi^{-} h_{c}$, $\pi^{+} \pi^{-} h_{c}(2 P), K^{+} K^{-} J / \psi, K^{+} K^{-} \psi(2 S), \pi^{+} \pi^{-} \psi(2 S), \pi^{+} \pi^{-} \psi_{2}, \omega \chi_{c J}, \phi \chi_{c J}$, etc., to search for more new vector charmoniumike states or new decay modes of the discovered $Y$ states.

\section{The $Z$ states}

After the charged charmoniumlike state $Z_{c}(3900)$ was observed by BESIII and Belle experiments [14, 22] BESIII and Belle observed a series of charged $Z_{c}$ states including $Z_{c}(4020)$ [19], $Z_{c}(4200)$ [23], and $Z_{c}(3885)$ [24]. These states seem to indicate that a new class of hadrons has been observed. As there are at least four quarks within these $Z_{c}$ states, they have been interpreted either as tetraquark states, molecular states, or other configurations. 
In the Belle updated $e^{+} e^{-} \rightarrow \pi^{+} \pi^{-} \psi(2 S)$ analysis [18] there is an excess evident at around $4.05 \mathrm{GeV} / c^{2}$ in the $\pi^{ \pm} \psi(2 S)$ invariant mass distributions in the $Y(4360)$ subsample defined as $4.0<M_{\pi^{+} \pi^{-} \psi(2 S)}<4.5 \mathrm{GeV} / c^{2}$. An unbinned maximum-likelihood fit is performed on the distribution of $M_{\max }\left(\pi^{ \pm} \psi(2 S)\right)$, the maximum of $M\left(\pi^{+} \psi(2 S)\right)$ and $M\left(\pi^{-} \psi(2 S)\right)$. The excess is parameterized with a Breit-Wigner function and the non-resonant non-interfering background with a secondorder polynomial function. The fit yields a mass of $\left(4054 \pm 3\right.$ (stat.) \pm 1 (syst.)) $\mathrm{MeV} / \mathrm{c}^{2}$, a width of $(45 \pm 11$ (stat.) \pm 6 (syst.) $) \mathrm{MeV}$ and a $3.5 \sigma$ significance. This evident structure is called $Z_{c}(4050)$, which is a possible excited state of the $Z_{c}(3900)$.

Two charged bottomoniumlike resonances, $Z_{b}(10610)$ and $Z_{b}(10650)$, have been observed by the Belle Collaboration in $e^{+} e^{-} \rightarrow \Upsilon(n \mathrm{~S}) \pi^{+} \pi^{-}, n=1,2,3$ and $e^{+} e^{-} \rightarrow h_{b}(m \mathrm{P}) \pi^{+} \pi^{-}, m=1,2$ [25, 26]. Several models suggested that the $Z_{b}(10610)$ and $Z_{b}(10650)$ states might be loosely bound $B \bar{B}^{*}$ and $B^{*} \bar{B}^{*}$ systems, respectively. If so, it is natural to expect the $Z_{b}$ states to decay to final states with $B^{(*)}$ mesons at substantial rates. Using a data sample with an integrated luminosity of $121.4 \mathrm{fb}^{-1}$ collected near the peak of the $\Upsilon(5 S)$ resonance, Belle reconstructed three-body $B^{(*)} \bar{B}^{(*)} \pi$ combinations with a charged primary pion [27], where the set of $B^{+} \bar{B}^{0} \pi^{-}$and $B^{-} B^{0} \pi^{+}$final states is referred to as $B B \pi$; the set of $B^{+} \bar{B}^{* 0} \pi^{-}, B^{-} B^{* 0} \pi^{+}, B^{0} B^{*-} \pi^{+}$and $\bar{B}^{0} B^{*+} \pi^{-}$final states is referred to as $B B^{*} \pi$; and the set of $B^{*+} \bar{B}^{* 0} \pi^{-}$and $B^{*-} B^{* 0} \pi^{+}$final states is denoted as $B^{*} B^{*} \pi$. $B$ mesons are reconstructed in 18 decay channels totally. There are $12263 \pm 168$ fully reconstructed $B$ mesons. In the missing mass distribution of $B \pi$, peaks corresponding to the $B B^{*} \pi$ and $B^{*} B^{*} \pi$ signals are evident. The $M_{\text {miss }}(\pi)$ distributions are shown in Fig. 3, where three models are used to fit: only the $Z_{b}(10610)\left[Z_{b}(10650)\right]$ amplitude [Model-0], an additional possible non-resonant component [Model-1], and a combination of two $Z_{b}$ amplitudes [Model-2]. The fit results are shown in Fig. 3. The fit to the $B B^{*} \pi$ data with Model- 0 gives $10605 \pm 6 \mathrm{MeV} / c^{2}$ and $25 \pm 7 \mathrm{MeV}$ for the $Z_{b}(10610)$ mass and width, respectively, and the fit to the $B^{*} B^{*} \pi$ data gives $10648 \pm 13 \mathrm{MeV} / c^{2}$ and $23 \pm 8 \mathrm{MeV}$ for the $Z_{b}(10650)$ mass and width, respectively.

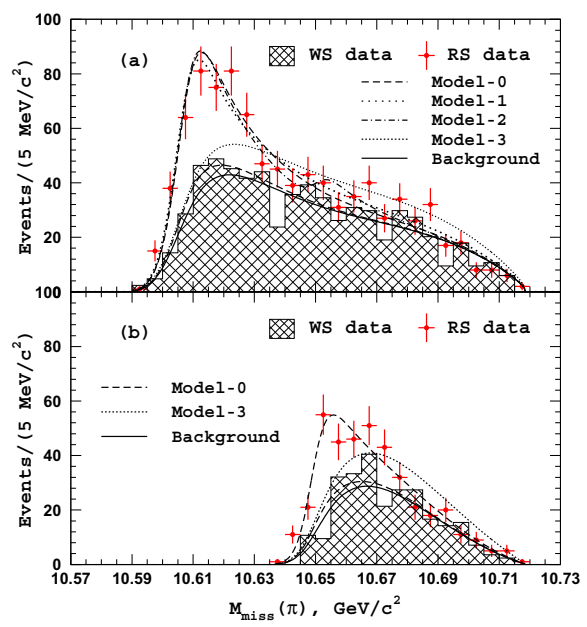

Figure 3. The $M_{\text {miss }}(\pi)$ distribution for the (a) $B B^{*} \pi$ and (b) $B^{*} B^{*} \pi$ candidate events.

The calculated ratio of the branching fractions are $\mathcal{B}\left(Z_{b}(10610) \rightarrow B \bar{B}^{*}+\right.$ c.c. $) / \mathcal{B}\left(Z_{b}(10610) \rightarrow\right.$ bottomonium $)=4.76 \pm 0.64 \pm 0.75$ and $\mathcal{B}\left(Z_{b}(10650) \rightarrow B^{*} \bar{B}^{*}\right) / \mathcal{B}\left(Z_{b}(10650) \rightarrow\right.$ bottomonium $)=$ $2.40 \pm 0.44 \pm 0.50$. Assuming that the $Z_{b}(10610)$ and $Z_{b}(10650)$ are saturated by the already observed $\Upsilon(n S) \pi, h_{b}(m \mathrm{P}) \pi$, and $B^{*} B^{(*)}$ channels, the decay rates are summarized in Table 1. 
Table 1. Branching fractions for the $Z_{b}^{+}(10610)$ and $Z_{b}^{+}(10650)$ decays.

\begin{tabular}{lcc}
\hline \hline Channel & \multicolumn{2}{c}{ Fraction, \% } \\
& $Z_{b}(10610)$ & $Z_{b}(10650)$ \\
\hline$\Upsilon(1 \mathrm{~S}) \pi^{+}$ & $0.60 \pm 0.17 \pm 0.07$ & $0.17 \pm 0.06 \pm 0.02$ \\
$\Upsilon(2 \mathrm{~S}) \pi^{+}$ & $4.05 \pm 0.81 \pm 0.58$ & $1.38 \pm 0.45 \pm 0.21$ \\
$\Upsilon(3 \mathrm{~S}) \pi^{+}$ & $2.40 \pm 0.58 \pm 0.36$ & $1.62 \pm 0.50 \pm 0.24$ \\
$h_{b}(1 \mathrm{P}) \pi^{+}$ & $4.26 \pm 1.28 \pm 1.10$ & $9.23 \pm 2.88 \pm 2.28$ \\
$h_{b}(2 \mathrm{P}) \pi^{+}$ & $6.08 \pm 2.15 \pm 1.63$ & $17.0 \pm 3.74 \pm 4.1$ \\
$B^{+} \bar{B}^{* 0}+\bar{B}^{0} B^{*+}$ & $82.6 \pm 2.9 \pm 2.3$ & - \\
$B^{*+} \bar{B}^{* 0}$ & - & $70.6 \pm 4.9 \pm 4.4$ \\
\hline \hline
\end{tabular}

Very recently Belle measured the energy dependence of the $e^{+} e^{-} \rightarrow h_{b}(n P) \pi^{+} \pi^{-}(n=1,2)$ cross sections from thresholds up to $11.02 \mathrm{GeV}$ using $121.4 \mathrm{fb}^{-1}$ of on-resonance $\Upsilon(5 S)$ data, as well as $1 \mathrm{fb}^{-1}$ of data taken at each of 19 different energies between 10.77 and $11.02 \mathrm{GeV}$ [28]. Clear $\Upsilon(10860)$ and $\Upsilon(11020)$ peaks with little or no continuum contribution are observed. The distributions of $M_{\text {miss }}(\pi)$ for the yields of $\pi^{+} \pi^{-} h_{b}(1 P)$ and $\pi^{+} \pi^{-} h_{b}(2 P)$ are not distributed uniformly in phase space; they populate the $Z_{b}(10610)$ and $Z_{b}(10650)$ mass region. The model with the $Z_{b}(10610)$ and $Z_{b}(10650)$ signals describes the data well: the confidence levels of the fits are $65 \%$ and $77 \%$ for the $h_{b}(1 P)$ and $h_{b}(2 P)$, respectively. The phase space hypothesis is excluded at the $3.6 \sigma$ and $4.5 \sigma$ levels in the $h_{b}(1 P) \pi^{+} \pi^{-}$and $h_{b}(2 P) \pi^{+} \pi^{-}$channels, respectively. The single $Z_{b}(10610)$ hypothesis is excluded at the $3.3 \sigma$ level in the $h_{b}(1 P) \pi^{+} \pi^{-}$channel, while the single $Z_{b}(10650)$ hypothesis cannot be excluded at a significant level.

\section{Other topics}

Using a $5.74 \mathrm{fb}^{-1}$ data sample collected at the peak of the $\Upsilon(1 S)$ resonance, containing $102 \times 10^{6}$ $\Upsilon(1 S)$ decays, Belle searched for some of the $X Y Z$ states in $\Upsilon(1 S)$ inclusive decays for the first time [29]. In these searches, fourteen decay modes are considered: $X(3872)$ and $Y(4260)$ to $\pi^{+} \pi^{-} J / \psi$; $Y(4260), Y(4360)$ and $Y(4660)$ to $\pi^{+} \pi^{-} \psi(2 S) ; Y(4260)$ to $K^{+} K^{-} J / \psi ; Y(4140)$ and $X(4350)$ to $\phi J / \psi$; $Z_{c}(3900)^{ \pm}, Z_{c}(4200)^{ \pm}$and $Z_{c}(4430)^{ \pm}$to $\pi^{ \pm} J / \psi ; Z_{c}(4050)^{ \pm}$and $Z_{c}(4430)^{ \pm}$to $\pi^{ \pm} \psi(2 S)$; and a predicted $Z_{c s}^{ \pm}$state with mass $(3.97 \pm 0.08) \mathrm{GeV} / c^{2}$ and width $(24.9 \pm 12.6) \mathrm{MeV}$ [30] to $K^{ \pm} J / \psi$. All the invariant mass distributions from a $J / \psi(\psi(2 S))$ and up to two charged light hadrons $\left(K^{ \pm} / \pi^{ \pm}\right)$are checked. No evident signal is found for any of them and $90 \%$ C.L. upper limits are set on the product branching fractions and listed in Table III in Ref. [29]. The tagged $J / \psi$ and $\psi(2 S)$ signals are observed clearly and the corresponding branching fractions are measured to be $\mathcal{B}(\Upsilon(1 S) \rightarrow J / \psi+$ anything $)=$ $(5.25 \pm 0.13$ (stat.) \pm 0.25 (syst. $)) \times 10^{-4}$ and $\mathcal{B}(\Upsilon(1 S) \rightarrow \psi(2 S)+$ anything $)=(1.23 \pm 0.17$ (stat. $) \pm$ 0.11 (syst.) $) \times 10^{-4}$ with substantially improved precision.

The LHCb collaboration reported the observation of two pentaquark states, denoted as $P_{c}(4380)^{+}$ and $P_{c}(4450)^{+}$, in the $J / \psi p$ system in $\Lambda_{b}^{0} \rightarrow J / \psi K^{-} p$ [31]. The first strong experimental evidence for a pentaquark state $\Theta(1540)^{+}$was reported in the reaction $\gamma n \rightarrow n K^{+} K^{-}$in the LEPS experiment [32]. However, it was not confirmed in larger-statistics data samples in the same experiment and was most probably not a genuine state. Very recently Belle did the first measurement $\gamma \gamma \rightarrow p \bar{p} K^{+} K^{-}$and searched for novel exotic baryons, denoted as $\Theta(1540)^{0} \rightarrow p K^{-}$and $\Theta(1540)^{++} \rightarrow p K^{+}$which are similar to $\Theta(1540)^{+}$, in intermediate processes [33]. The results are based on an analysis of a $980 \mathrm{fb}^{-1}$ data sample taken at or near the $\Upsilon(n S)(n=1, \ldots, 5)$ resonances with the Belle detector. Clear $\gamma \gamma \rightarrow p \bar{p} K^{+} K^{-}$signals are observed and the corresponding cross section is measured. No clear 
structure is seen in the corss section distribution and the largest value is around $40 \mathrm{pb}$, which is lower than the theoretical rough estimate of $100 \mathrm{pb}$. While the $\Lambda(1520)^{0}$ signals in $p K^{-}$invariant mass spectrum are also observed, no evidence for any exotic baryon is seen in the $p K^{-}$or $p K^{+}$invariant mass spectrum. For all of the above-mentioned processes, the cross sections are measured for the first time.

Supported in part by the National Natural Science Foundation of China under Contract No. 11575017 and National Key Basic Research Program of China under Contact No. 2015CB856701.

\section{References}

[1] N. Brambilla et al., Eur. Phys. J. C 71, 1534 (2011)

[2] S. Godfrey and S. L. Olsen, Annu. Rev. Nucl. Part. Sci. 58, 51 (2008)

[3] N. Brambilla et al., Eur. Phys. J. C 74, 2981 (2014)

[4] C. Z. Yuan, Int. J. Mod. Phys. A 29, 1430046 (2014)

[5] S. K. Choi et al. (Belle Collaboration), Phys. Rev. Lett. 91, 262001 (2003)

[6] M. Ablikim et al. (BESIII Collaboration), Phys. Rev. Lett. 112, 092001 (2014)

[7] S. Chatrchyan et al. (CMS Collaboration), Phys. Lett. B 727, 57 (2013)

[8] X. H. He et al. (Belle Collaboration), Phys. Rev. Lett. 113, 142001 (2014)

[9] B. Aubert et al. (BaBar Collaboration), Phys. Rev. Lett. 95, 142001 (2005)

[10] Q. He et al. (CLEO Collaboration), Phys. Rev. D 74, 091104(R) (2006)

[11] K. Abe et al. (Belle Collaboration), hep-ex/0612006

[12] C. Z. Yuan et al. (Belle Collaboration), Phys. Rev. Lett. 99, 182004 (2007)

[13] J. P. Lees et al. (BaBar Collaboration), Phys. Rev. D 86, 051102(R) (2012)

[14] Z. Q. Liu et al. (Belle Collaboration), Phys. Rev. Lett. 110, 252002 (2013)

[15] B. Aubert et al. (BaBar Collaboration), Phys. Rev. Lett. 98, 212001 (2007)

[16] X. L. Wang et al. (Belle Collaboration), Phys. Rev. Lett. 99, 142002 (2007)

[17] B. Aubert et al. (BaBar Collaboration), Phys. Rev. D 89, 111103 (2014)

[18] X. L. Wang et al. (Belle Collaboration), Phys.Rev. D 91, 112007 (2015)

[19] M. Ablikim et al. (BESIII Collaboration), Phys. Rev. Lett. 111, 242001 (2013)

[20] M. Ablikim et al. (BESIII Collaboration), Phys. Rev. Lett. 114, 092003 (2015)

[21] M. Ablikim et al. (BESIII Collaboration), Phys. Rev. Lett. 115, 011803 (2015)

[22] M. Ablikim et al. (BESIII Collaboration), Phys. Rev. Lett. 110, 252001 (2013)

[23] K. Chilikin et al. (Belle Collaboration), Phys. Rev. D 90, 112009 (2014)

[24] M. Ablikim et al. (BESIII Collaboration), Phys. Rev. Lett. 112, 022001 (2014)

[25] A. Bondar et al. (Belle Collaboration), Phys. Rev. Lett. 108, 122001 (2012)

[26] A. Garmash et al. (Belle Collaboration), Phys. Rev. D 91, 072003 (2015)

[27] A. Garmash et al. (Belle Collaboration), Phys. Rev. Lett. 116, 212001 (2016)

[28] R. Mizuk et al. (Belle Collaboration), Phys. Rev. Lett. 117, 142001 (2016)

[29] C. P. Shen et al. (Belle Collaboration), Phys. Rev. D 93, 112013 (2016)

[30] S. H. Lee, M. Nielsen and U. Wiedner, J. Korean Phys. Soc. 55, 424 (2009); J. M. Dias, X. Liu and M. Nielsen, Phys. Rev. D 88, 096014 (2013)

[31] R. Aaij et al. (LHCb Collaboration), Phys. Rev. Lett. 115, 072001 (2015)

[32] T. Nakano et al. (LEPS Collaboration), Phys. Rev. Lett. 91, 012002 (2003)

[33] C. P. Shen et al. (Belle Collaboration), Phys. Rev. D 93, 112017 (2016) 